\title{
Editorial
}

\section{The challenge of early gastric cancer: the need to optimize microstaging and therapy}

\author{
Harold J. WANEBO \\ Department of Surgery, Roger Williams Medical Center (affiliated with Boston University School of Medicine), 825 Chalkstone Avenue, \\ Providence, Rhode Island 02908, USA
}

The term, "early gastric cancer (EGC)," defined as carcinoma limited to the gastric mucosa and submucosa in 1963 by the Japanese Gastroenterology and Endoscopy Society, has continued to spark controversy over the years [1-3]. Earlier descriptions of the entity include those by several authors, ranging from Haus of Leipzig, in 1883; Verse of Leipzig, in 1908; and Saeki of Tokyo, in 1938; to Stout, in 1942; however, the use of this term in the diagnosis and management of gastric cancer has recently taken on a new reality [2]. The incidence of EGC has increased, especially in Japanese series, to perhaps $30 \%-40 \%$ of invasive gastric cancers. A recent study from Korea (Kim et al. [4]) reviewed 1136 patients with EGC, which constituted approximately $15 \%$ of their total series of 7606 gastric cancer patients. In their review of nine clinical pathologic features associated with EGC, Kim and colleagues reported that the key factors linked with node metastases and risk of death were depth of invasion and macroscopic type (protruding types I and II). Over $15.7 \%$ of the ECG group had lymph node metastases.

Numerous studies (largely Japanese) have focused on key parameters that could be associated with the risk of nodal metastases or treatment failure in EGC [2,3]. A major factor appearred to be depth of invasion. There was a very clear relationship between depth of invasion and the incidence of nodal metastases, which ranged from $2 \%$ with mucosal invasion to $19.6 \%$ with submucosal invasion. The incidence rose to very high levels $(36.7 \%)$ with the presence of muscularis invasion, and was up to $60 \%$ with subserosal involvement [2]. In the study of Sano et al. [3], tumor diameter was also important. The risk of lymph node metastasis was higher in patients with lesions of more than $5-\mathrm{cm}$ diameter than in those with lesions $3-3.8 \mathrm{~cm}$ in diameter. Tumors

Offprint requests to: H.J. Wanebo

Received: November 10, 2000 / Accepted: November 13, 2000 considered suitable for local resection included those confined to the mucosa (less than $1.5 \mathrm{~cm}$ ), those that were macroscopically elevated, and those that were depressed without intramural ulcers or ulcer scars [3].

The impact of nodal metastases in early lesions is well demonstrated in the study of Abe et al. [2], in which the survival of patients with either T1N0 or T2N0 cancer was the same $(100 \%)$, in contrast to the reduced survival in those having nodal metastases (T1N1 or T2N1), in whom the survival rate was $72 \%-74 \%$ [2]. In that series, Abe and colleagues recommended lymphadenectomy with secondary or tertiary lymph node dissection, depending on the primary invasive level of the EGC. Inoue et al. [5], in an informative study, reported on the impact of depth of invasion on the frequency of nodal metastases and on outcome. The 5-year survival was $100 \%$ in patients with mucosal lesions and $90 \%$ in those with submucosal invasion. Lymph node metastases exerted a major impact on survival that ranged from an overall survival of $99 \%$, if N0, to $73 \%$, if N1. In patients with mucosal lesions there was a $4 \%$ incidence of nodal metastases, which were primarily confined to N1 nodes. In patients with submucosal lesions, half of the nodal metastases involved N2 nodes and even an occasional N3 node. This finding suggested that there was a step-wise progression of micrometastases with invasion. The recommendation of Inoue and colleagues was endoscopic removal for mucosal lesions, with an $\mathrm{N} 1$ dissection as a consideration. In patients with submucosal lesions, it was recommended to perform a D2 dissection along with subtotal gastrectomy.

The major problem in microstaging appears to be a lack of agreement regarding the characteristics that truly classify nonthreatening EGC, which has excellent survival rates, in the $98 \%-100 \%$ range, versus more threatening lesions that have a lymph node metastatic incidence in the $15 \%-20 \%$ range and survival rates of $69 \%-70 \%$. This issue is brought into focus in the study reported by Saragoni et al. [6] in this issue of the 
Journal. These authors based their microstage schema on the classifications described by Kodama et al. [6,7]. Kodama and colleagues utilized a scheme that coupled the invasiveness of the EGC with its macroscopic characteristics; their major types were: small mucosal (SM) type $(36.5 \%)$, superficial spreading (Super M) type $(44.9 \%)$, and a penetrating growth pattern (Pen) type $(17.4 \%)$ that invaded the submucosa [7]. The Pen type was further divided into a Pen A subtype that grew expansively into the submucosa and a Pen B subtype that grew deeper, penetrating the submucosa. The SM, Super M, and Pen B types had a low incidence of vessel invasion and lymph node metastasis and had an excellent prognosis (more than 90\% 5-year survival rate) in contrast to the outcome in patients with the Pen A type. Pen A lesions were characterized by a dominant expansive lesion, invading the submucosa, and containing a well differentiated cancer, with high incidences of vessel invasion and lymph node metastases, and poor prognosis (64.8\% 5-year survival rate); these was also a high incidence of liver metastases [7].

This staging system of Kodama and colleagues [7] was used as the basis for microstaging in the Italian patient series reported by Saragoni et al. [6]. Utilizing this system, they characterized the lesions in a group of more than 412 EGC patients whose overall survival at 5 and 10 years was $93 \%$ and $89 \%$, respectively. A lower survival was shown to be related to invasion into the submucosa (Pen A type) and was also shown in patients that had lymph node metastases. A bivariate analysis showed that the worst prognosis was associated with the Pen A type, SM invasion, and lymph node metastases. The Pen A type, per se, had a $58 \%$ incidence of nodal metastases (32 of 55 patients) and a survival rate of $74 \%$, versus a $7.6 \%$ incidence of nodal metastases (23 of 302 patients) and $96 \%$ survival in the non-Pen A patients. The risk of death hazard ratio of 6.54 suggests strong biologic behavior for the Pen A type growth pattern.

The Italian group of Saragoni et al. [6] is one of the few Western series to examine the issue of EGC utilizing the Kodama staging system to characterize these lesions. In addition to depth of invasion, they have confirmed an important added prognostic feature of Pen A, which is a growth pattern that appears to represent a risk feature independent of invasion, per se, and which has a strong correlation with nodal metastases and impaired survival.
How can one make use of this finding? The controversial questions raised by numerous Japanese authors and this recent Italian study suggest that EGC microstaging is an important concept, which has long been recognized by Japanese authorities and is now being recognized in Western studies. Thus, there seems to be a need for a clinical trial to examine the issue. Such a clinical trial might examine the issue of optimum staging parameters that certainly should include tumor invasiveness of the mucosa and submucosa, tumor size, and the macroscopic characteristics described by Kodama et al. [7] and others. The issue of endoscopic excision for mucosal lesions, only, with planned limited gastrectomy $[8,9]$, and node dissection for submucosal lesions or high-risk lesions, described as Pen A type by Kodama et al. [7], or described as large cancers (more than $5 \mathrm{~cm}$ ) by Sano et al. [3] could be examined. It is only with clinical trials that the issues of proper microstaging (i.e., as with melanoma) and optimum therapy in EGC patients can be addressed.

\section{References}

1. Japanese Research Society for Gastric Cancer. The general rules for gastric cancer study in surgery and pathology. Jpn J Surg 1981;11:127-39.

2. Abe S, Yoshimura H, Nagaoka S, Monden N, Kinugasa S, Nagasue $\mathrm{N}$, et al. Long-term results of operation for carcinoma of the stomach in T1/T2 stages: critical evaluation of the concept of early carcinoma of the stomach. J Am Coll Surg 1995;181:389-96.

3. Sano T, Kobori O, Muto T. Lymph node metastasis from gastric cancer: endoscopic resection of tumour. Br J Surg 1992:79:241-4.

4. Kim JP, Hur YS, Yang HK. Lymph node metastasis as a significant prognostic factor in early gastric cancer: analysis of 1136 early cancers. Ann Surg Oncol 1995;2:308-13.

5. Inoue K, Tobe T, Kan N, Nio Y, Sakai M, Takeuchi E, et al. Problems in the definition and treatment of early gastric cancer. $\mathrm{Br}$ J Surg 1991;78:818-21.

6. Saragoni L, Gaudio M, Morgagni P, Folli S, Vio A, Scarpi E, Saragoni A. The role of growth patterns according to Kodama's classification and lymph node status as independent prognostic factors in early gastric cancer: analysis of 412 cases. Gastric Cancer 2000;3:134-40.

7. Kodama Y, Inokuchi K, Soejima K, Matsusaka K, Okamura T. Growth patterns and prognosis in early gastric cancer: superficially spreading and penetrating growth types. Cancer 1983;51:320-6.

8. Arima N, Adachi K, Katsube T, Amano K, Ishihara S, Watanabe M, Kinoshita Y. Predictive factors for metachronous recurrence of early gastric cancer after endoscopic treatment. J Clin Gastroenterol 1999;29:44-7.

9. El-Zamaity HMT, Ota H. Endoscopic resection for early gastric cancer: possibilities and limitations. J Clin Gastroenterol 1999;29: $5-6$. 